\title{
SUBSTRUCTURE PRECONDITIONERS FOR ELLIPTIC SADDLE POINT PROBLEMS
}

\author{
TORGEIR RUSTEN AND RAGNAR WINTHER
}

\begin{abstract}
Domain decomposition preconditioners for the linear systems arising from mixed finite element discretizations of second-order elliptic boundary value problems are proposed. The preconditioners are based on subproblems with either Neumann or Dirichlet boundary conditions on the interior boundary. The preconditioned systems have the same structure as the nonpreconditioned systems. In particular, we shall derive a preconditioned system with conditioning independent of the mesh parameter $h$. The application of the minimum residual method to the preconditioned systems is also discussed.
\end{abstract}

\section{INTRODUCTION}

The purpose of this paper is to propose domain decomposition techniques for elliptic saddle point problems. Here, elliptic saddle point problems refers to the discrete systems resulting from mixed finite element discretizations of secondorder elliptic boundary value problems. We study a preconditioned iterative method for these systems where a decomposition of the domain into simpler substructures is utilized in order to construct the preconditioners.

Let $\Omega \subset \mathbb{R}^{2}$ be a polygonal domain, and let $\partial \Omega$ denote the boundary. We consider the Dirichlet problem

$$
\begin{aligned}
-\nabla \cdot K(x) \nabla p & =f & & \text { in } \Omega, \\
p & =g & & \text { on } \partial \Omega,
\end{aligned}
$$

where $f$ and $g$ are given functions. The matrix $K(x)$ is assumed to be symmetric and uniformly positive definite on $\Omega$.

If this boundary value problem is discretized by a conforming finite element method, we obtain a linear system with a symmetric and positive definite coefficient matrix. However, the coefficient matrix is not well conditioned. If the dimension of the system is sufficiently large, the system has to be solved by an iterative method. In order to obtain a well-conditioned system, and hence fast convergence of the iterative method, preconditioning of the system is necessary. The behavior of an iterative method, therefore, depends on the construction of easily invertible preconditioners.

Received by the editor September 13, 1991.

1991 Mathematics Subject Classification. Primary 65F10, 65N30.

Key words and phrases. Second-order elliptic equations, mixed finite element methods, domain decomposition.

This research has been supported by VISTA, a research cooperation between the Norwegian Academy of Science and Letters and Den norske stats oljeselskap a.s. (STATOIL).

(C) 1993 American Mathematical Society $0025-5718 / 93 \$ 1.00+\$ .25$ per page 
The most common iterative method for the discrete systems arising from a conforming finite element discretization of boundary value problems of the form (1.1) is the preconditioned conjugate gradient method. The convergence properties of the basic iterative method is well understood, and the construction of preconditioners has been intensively studied. This has resulted in very effective iterative methods for such systems.

The use of incomplete factorization as preconditioners for discrete elliptic equations is a well-established technique. One advantage with these preconditioners is that they are usually very easy to implement. Furthermore, both theory and experiments show that these preconditioners can be rather effective. For a more precise description of these preconditioners and their performance we refer, for example, to $[1,12,23]$.

Another approach to effective preconditioners is based on substructuring or domain decomposition. The main idea behind these constructions is to decompose the domain $\Omega$ into simpler substructures such that certain discrete elliptic systems can be solved by a fast solver on these subdomains. The complete preconditioner is then constructed by a proper composition of these fast subsolvers. From a theoretical point of view these sophisticated constructions are very attractive, since one may obtain convergence rates independent of the number of unknowns. As a consequence, the work required to obtain a certain accuracy will essentially be proportional to the number of unknowns. We refer to $[3,4,6,7,21]$, and references given there, for a more detailed discussion of domain decomposition methods applied to the discrete equations arising from a conforming finite element method.

Even though a conforming finite element discretization seems to be the obvious approach to second-order elliptic boundary value problems of the form (1.1), there are applications where the discretization by a mixed finite element method may be desirable. In some problems the gradient of the solution is the variable of primary interest. This is the case, for example, for the pressure equation in the coupled system modeling incompressible two-phase flow in porous media (cf., e.g., [2]). In this case, $p$ is the pressure, $K$ corresponds to the mobility matrix, and the most important variable is the Darcy velocity given by $-K \nabla p$. If a conforming finite element method is used to discretize the pressure equation, the Darcy velocity is derived by performing a numerical differentiation on the computed pressure. Hence, some of the accuracy of the numerical solution is lost. On the other hand, if a mixed finite element method is used to discretize the pressure equation, the pressure and the Darcy velocity can be computed simultaneously from the discrete system, and with the same degree of accuracy. The use of a mixed finite element method for the discretization of the pressure equation has therefore been suggested by many authors, $\mathrm{cf}$. $[13,15,26]$.

The discretization of the elliptic boundary value problem (1.1) by the mixed finite element method leads to a discrete system with a saddle point structure of the form

$$
\begin{aligned}
A \xi+B \eta & =b, \\
B^{T} \xi & =c .
\end{aligned}
$$

Here, $A \in \mathbb{R}^{m \times m}$ is symmetric and positive definite, $B \in \mathbb{R}^{m \times n}$ with $n \leq m$, 
and $B$ has full rank, i.e., $\operatorname{rank}(B)=n$. It is well known that systems of the form (1.2) have a unique solution $\xi \in \mathbb{R}^{m}$ and $\eta \in \mathbb{R}^{n}$. Furthermore, the coefficient matrix $\mathscr{A}$ of system (1.2), given by

$$
\mathscr{A}=\left(\begin{array}{cc}
A & B \\
B^{T} & 0
\end{array}\right) \text {, }
$$

is symmetric, nonsingular, and indefinite.

Since the coefficient matrix of the discrete system is indefinite, the construction of effective iterative methods for the discrete system (1.2) is not as well studied as for systems arising from a conforming finite element method. However, if the matrix $A$ can be easily inverted, then the system (1.2) can be essentially reduced to two positive definite systems by a block elimination procedure. The variable $\eta$ satisfies the system

$$
B^{T} A^{-1} B \eta=B^{T} A^{-1} b-c .
$$

If we first compute $\eta$ from (1.4), then the variable $\xi$ can thereafter be obtained from the first equation of (1.2). Hence, in this case standard iterative methods for positive definite systems can be applied. We refer for example to [14, 17] for this approach to the solution of linear systems obtained from mixed finite element discretizations of systems of the form (1.1).

However, in many practical computations the matrix $A$ cannot be easily inverted. For example, this is usually the case for the pressure equation arising in the modeling of flow in porous media, when the mobility matrix $K$ is nondiagonal. In such cases the equation (1.4) has to be solved by an iterative method with an inner and an outer iteration. As illustrated in [27], such two-level methods may be numerically unstable. In order to avoid such problems, it seems to be more attractive to design a preconditioned iterative method directly for the symmetric, indefinite system (1.2). Such an approach is discussed in [27]. The basic iterative method is the minimum residual method, which was first proposed by Paige and Saunders [24] for general symmetric systems. In addition, the block structure of the saddle point problems (1.2) is utilized in order to construct effective preconditioners.

We should mention here that an alternative approach to the design of iterative methods for systems of the form (1.2) is discussed in [5]. The methods considered there are derived from a positive definite reformulation of the system. However, in the present paper we shall only consider the preconditioned minimum residual method developed in [27].

As established in [27], the convergence rate of the minimum residual method applied to a system of the form (1.2) is dominated by three parameters. These are the condition numbers of $A$ and $B$, and a third parameter measuring the relative scaling between them. Hence, if $A$ and $B$ are properly scaled, the purpose of an effective preconditioner is to improve the conditioning of each of the two matrices.

When the system (1.2) is derived from mixed finite element discretization of second-order elliptic equations of the form (1.1), the condition number of the matrix $A$ is dominated by the behavior of the coefficient matrix $K$ (cf. $\S 2$ ). Hence, if $K$ is well conditioned uniformly in $x$, then $A$ is also well conditioned. Therefore, the purpose of a preconditioner for this system is to 
improve the conditioning of $B$. However, $B$ is a discrete gradient operator and $B^{T} B$ is a discrete Laplacian. A preconditioner for the matrix $B$, and hence a complete preconditioner for (1.3), can therefore be derived from a suitable preconditioner for a discrete Laplace operator.

In [27] the preconditioned minimum residual method is applied, in particular, to the discrete systems obtained from mixed finite element discretizations of second-order elliptic problems of the form (1.1). The preconditioners studied there were constructed either by incomplete factorization procedures or by the use of fast solvers for an associated constant-coefficient problem on the domain $\Omega$. The purpose of the present paper is to extend this study to the applications of preconditioners constructed by fast solvers associated with the different substructures of the domain, i.e., to preconditioners constructed by domain decomposition.

It is appropriate to mention here that Glowinski and Wheeler [19] and Mathew [22] have previously studied preconditioning by domain decomposition for the discrete systems obtained by mixed finite element formulations of problems like (1.1). However, their approach requires that the discrete system can be solved exactly by a fast direct method on the subdomains. By these subdomain solvers the discrete saddle point problem is reduced to a symmetric, positive definite system on the interior boundary, and hence the preconditioned conjugate gradient method can be applied. However, for variable-coefficient problems it will usually not be possible to solve the subdomain problems exactly. Therefore, such a reduction of the system to positive definite form is not possible.

The approach taken in this paper only requires that a fast solver exists for certain related (constant-coefficient) problems on each subdomain. These subsolvers are then used to construct preconditioners for the discrete system, i.e., to transform the system into a new system of the form (1.2), but where the proper condition number of the matrix $B$ is reduced.

In $\S 2$ we give a brief review of the mixed finite element method for elliptic boundary value problems of the form (1.1). In $\S 3$ we state the assumptions that will be made on the domain $\Omega$ and on the finite element spaces, while the general formulation of the preconditioned minimum residual method for saddle point problems is reviewed in $\S 4$. The domain decomposition preconditioners are presented in $\S \S 5$ and 6 . In $\S 5$ we study a preconditioner which is based on subproblems with Neumann boundary condition on the interior boundary. We establish that this method leads to a reduction in the proper condition number from $O\left(h^{-1}\right)$ to $O\left(h^{-1 / 2}\right)$. Here the parameter $h$ corresponds to the grid size. As an alternative, we study in $\S 6$ a preconditioner based on subproblems with Dirichlet boundary condition. This method can be considered as a mixed analog of the method studied by Bramble, Pasciak, and Schatz $[6,7]$ for conforming finite elements (cf. also Bjørstad and Widlund [3]). A main tool in the techniques developed in [7] is to utilize the fact that a decomposition of the system into Dirichlet problems on the subdomains corresponds to an orthogonal decomposition of the solution. We derive a similar property for a generalized mixed finite element solution. From this property we design a preconditioner which is optimal (i.e., the condition number is independent of h) also for problems with variable coefficients. Finally, in $\S 7$ we present some numerical experiments which confirm our theoretical results. 


\section{THE MIXED FINITE ELEMENT METHOD}

In this section we give a brief review of the mixed finite element method for elliptic boundary value problems of the form (1.1). For a more detailed discussion of this topic we refer to $[8,16,25]$.

We recall that the matrix $K(x) \in \mathbb{R}^{2 \times 2}$ is assumed to be bounded and uniformly positive definite on $\Omega$, i.e., there exist positive constants $\tau_{0}$ and $\tau_{1}$ such that the inequalities

$$
\tau_{0}|\xi|^{2} \leq K(x) \xi \cdot \xi \leq \tau_{1}|\xi|^{2}
$$

hold for all $x \in \Omega$ and for all $\xi \in \mathbb{R}^{2}$, where $|\cdot|$ denotes the Euclidean norm on $\mathbb{R}^{2}$.

The mixed finite element method is derived from a reformulation of the equation (1.1), where the function $u=-K \nabla p$ is introduced as a new unknown variable. The elliptic equation (1.1) can then be rewritten as a system consisting of the equations

$$
\begin{aligned}
u+K \nabla p & =0, \\
\nabla \cdot u & =f,
\end{aligned}
$$

in $\Omega$, together with the boundary condition

$$
p=g \text { on } \partial \Omega .
$$

In order to give a precise formulation of this system, we need some notation. We will use $(\cdot, \cdot)$ to denote the inner products on $L^{2}(\Omega)$ and $\|\cdot\|$ to denote the corresponding norm. For convenience, we also use the same notation for the norm and inner product on the product space $\left(L^{2}(\Omega)\right)^{2}$. Furthermore, $\|\cdot\|_{\text {div }}$ will denote the norm on $H(\operatorname{div}, \Omega)$. Here, $H(\operatorname{div}, \Omega) \subset\left(L^{2}(\Omega)\right)^{2}$ is the space of all vectors $v \in\left(L^{2}(\Omega)\right)^{2}$ such that $\nabla \cdot v \in L^{2}(\Omega)$, and the norm is given by

$$
\|v\|_{\text {div }}^{2}=\|v\|^{2}+\|\nabla \cdot v\|^{2} \text {. }
$$

The normal component on the boundary $\partial \Omega$ of a function $v \in H(\operatorname{div}, \Omega)$ is denoted by $v \cdot n_{0}$, where $n_{0}$ is the unit outward normal to $\partial \Omega$. It is well known that this normal trace operator is a continuous operator from $H(\operatorname{div}, \Omega)$ into the Sobolev space $H^{-1 / 2}(\partial \Omega)$. The space $H^{-1 / 2}(\partial \Omega)$ is the dual space of the fractional Sobolev space $H^{1 / 2}(\partial \Omega)$ of functions defined on the boundary $\partial \Omega$. We denote by $|\cdot|_{-1 / 2, \partial \Omega}$ and $|\cdot|_{1 / 2, \partial \Omega}$ the norms on these boundary spaces, and by $\langle\cdot, \cdot\rangle_{\partial \Omega}$ the duality pairing between them. We refer to [18] for more details on the different function spaces introduced above. The given functions $f$ and $g$ in (1.1) are supposed to be in $L^{2}(\Omega)$ and $H^{1 / 2}(\partial \Omega)$, respectively. The usual mixed formulation of (1.1) now reads:

Find $(u, p) \in H(\operatorname{div}, \Omega) \times L^{2}(\Omega)$ such that

$$
\begin{aligned}
a(u, v)+b(v, p) & =G(v) \quad \forall v \in H(\operatorname{div}, \Omega), \\
b(u, q) & =F(q) \quad \forall q \in L^{2}(\Omega) .
\end{aligned}
$$

Here, the bilinear forms $a: H(\operatorname{div}, \Omega) \times H(\operatorname{div}, \Omega) \mapsto \mathbb{R}$ and $b: H(\operatorname{div}, \Omega) \times$ $L^{2}(\Omega) \mapsto \mathbb{R}$ are defined by

$$
a(v, w)=\left(K(x)^{-1} v, w\right) \text { and } b(v, q)=-(\nabla \cdot v, q),
$$

and the linear functionals $F: L^{2}(\Omega) \mapsto \mathbb{R}$ and $G: H(\operatorname{div}, \Omega) \mapsto \mathbb{R}$ are defined by

$$
F(q)=-(f, q) \text { and } G(v)=-\left\langle g, v \cdot n_{0}\right\rangle_{\partial \Omega} .
$$


This is an example of a variational problem with a saddle point structure. Such problems are discussed, for example, in $[8,18]$. From the general theory of such problems it follows that there exists a unique solution $(u, p)$ of $(2.2)$. Note that in this formulation the Dirichlet boundary condition $p=g$ on $\partial \Omega$ is a natural boundary condition. As a consequence, the boundary condition is fulfilled only in the weak sense.

The variational formulation (2.2) is the basis for the formulation of the mixed finite element method for (1.1). In order to approximate $u$ and $p$, we choose finite-dimensional subspaces $V=V_{h} \subset H(\operatorname{div}, \Omega)$ and $Q=Q_{h} \subset L^{2}(\Omega)$. Here, $h \in(0,1]$ is a discretization parameter, typically taken to be a measure of the size of the elements generating the spaces $V$ and $Q$. The approximation $\left(u_{h}, p_{h}\right)$ of $(u, p)$ is required to be an element of the space $V \times Q$. The spaces $V$ and $Q$ are typically taken to be finite element spaces, constructed from basis functions which are piecewise polynomials. For details on the construction of suitable finite element spaces $V$ and $Q$ for a general domain $\Omega$ we refer to $[9,10,11,25]$.

When the spaces $V$ and $Q$ are constructed, the approximation $\left(u_{h}, p_{h}\right) \in$ $V \times Q$ is determined by the linear system

$$
\begin{aligned}
a\left(u_{h}, v\right)+b\left(v, p_{h}\right) & =G(v) \quad \forall v \in V, \\
b\left(u_{h}, q\right) & =F(q) \quad \forall q \in Q .
\end{aligned}
$$

If the basis functions for the finite element spaces $V$ and $Q$ are introduced, this system is exactly of the form (1.2).

However, in order to obtain a stable numerical method, the spaces $V$ and $Q$ have to be properly balanced. This is expressed by a so-called inf-sup condition, i.e., there exists a constant $\gamma$, independent of $h$, such that

$$
\inf _{q \in Q} \sup _{v \in V} \frac{b(v, q)}{\|q\|\|v\|_{\text {div }}} \geq \gamma>0 .
$$

Roughly, this condition expresses that if the "pressure space" $Q$ has been chosen, then the "velocity space" $V$ has to be taken sufficiently large. In addition, the spaces $V$ and $Q$ have to be chosen such that

$$
\sup _{q \in Q} b(v, q)>0
$$

for all $v \in V$ with $\nabla \cdot v \neq 0$. The two conditions (2.5) and (2.6) are sufficient to guarantee the stability of the mixed finite element method. In particular, the discrete system (2.4) has a unique solution. Furthermore, the numerical solution $\left(u_{h}, p_{h}\right)$ satisfies a stability estimate of the form (cf. $\left.[8,25]\right)$

$$
\left\|u_{h}\right\|_{\text {div }}+\left\|q_{h}\right\| \leq c\left(\|u\|_{\text {div }}+\|q\|\right),
$$

where $(u, p)$ denotes the corresponding solution of $(2.2)$ and $c$ is a constant independent of the solutions and the discretization parameter $h$.

In order to construct preconditioners for the discrete systems by domain decomposition, the spaces $V$ and $Q$ also have to be properly related to the decomposition of the domain. These conditions on the finite element spaces will be discussed in $\S 3$.

\section{Domain DECOMPOSITION}

In this section we first describe the decomposition properties of the domain $\Omega$. We will also specify the required assumptions on the finite element spaces 


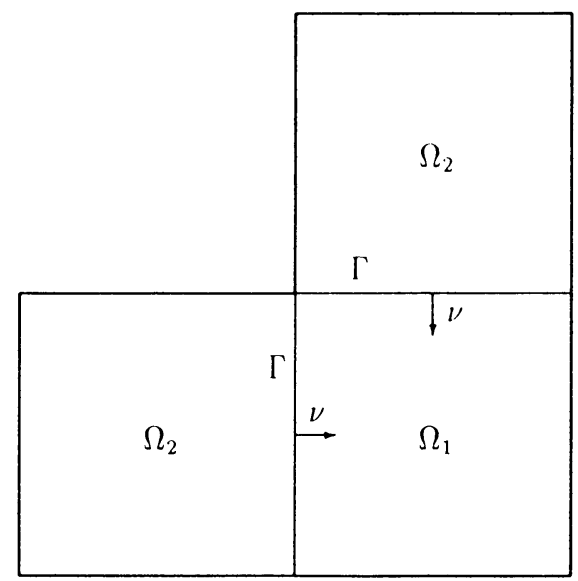

FIGURE 3.1. An L-shaped domain $\Omega$ divided into two subdomains

$V$ and $Q$. These assumptions, which in particular imply the desired conditions (2.5) and (2.6), will be utilized later in order to construct preconditioners for the discrete saddle point problem (2.4). In $\S 7$ we will present some examples where all the assumptions on $V$ and $Q$ given here will be verified.

Throughout this paper, we will assume that the domain $\Omega$ is a union of two nonoverlapping subdomains, denoted by $\Omega_{1}$ and $\Omega_{2}$, with a common boundary $\Gamma$. Furthermore, we also assume that both $\partial \Omega_{1} \cap \partial \Omega$ and $\partial \Omega_{2} \cap \partial \Omega$ have positive measures. Here, $\partial \Omega_{i}$ denotes the boundary of $\Omega_{i}$, and we assume that $\Gamma$ consists of one or more line segments with both endpoints on $\partial \Omega$. An example of a domain, divided into subdomains as specified above, is given in Figure 3.1. Examples of more complex regions are given, e.g., in [3, 7].

The preconditioners studied below will be constructed from exact solvers of related discrete elliptic problems on the subdomains $\Omega_{i}$. A basic implicit assumption is therefore that the subdomains have a simple geometry such that these elliptic subsolvers are sufficiently fast.

In addition to the function spaces introduced in $\S 2$ above, we will also need some spaces related to the interior boundary $\Gamma$. The space of square integrable functions with respect to the arc length of $\Gamma$ will be denoted by $L^{2}(\Gamma)$, and $\langle\cdot, \cdot\rangle_{\Gamma}$ is the associated inner product. If $H^{1}(\Gamma)$ is the corresponding firstorder Sobolev space on $\Gamma$, we let

$$
H_{0}^{1}(\Gamma)=\left\{\varphi \in H^{1}(\Gamma): \varphi=0 \text { at } \partial \Omega\right\} .
$$

Furthermore, $H_{0}^{1 / 2}(\Gamma)$ will denote the interpolation space halfway between $L^{2}(\Gamma)$ and $H_{0}^{1}(\Gamma)$. (This space is frequently denoted $H_{0,0}^{1 / 2}(\Gamma)$, c.f. [6] or [20].) The dual space of $H_{0}^{1 / 2}(\Gamma)$ with respect to $\langle\cdot, \cdot\rangle_{\Gamma}$ will be denoted $H^{-1 / 2}(\Gamma)$. The norm on the space $H^{\theta}(\Gamma)$ is denoted by $|\cdot|_{\theta, \Gamma}$, where the subscript $\theta$ is dropped if $\theta=0$.

The unit normal vector on the interior boundary $\Gamma$, exterior to the subdomain $\Omega_{i}$, will be denoted $\nu_{i}$ for $i=1,2$. If $v \in H\left(\operatorname{div}, \Omega_{i}\right)$, then $v \cdot \nu_{i}$ will denote the corresponding scalar function on $\Gamma$. Here the values of $v$ on $\Gamma$ are 
derived from the domain $\Omega_{i}$. We recall from [18] that the map

$$
v \mapsto v \cdot \nu_{i}
$$

is continuous as a map from $H\left(\operatorname{div}, \Omega_{i}\right)$ into $H^{-1 / 2}(\Gamma)$. If $v \in H\left(\operatorname{div}, \Omega_{1}\right) \oplus$ $H\left(\operatorname{div}, \Omega_{2}\right)$, we let $[v \cdot \nu]$ be the jump of these traces, i.e.,

$$
[v \cdot \nu]=v \cdot \nu_{2}+v \cdot \nu_{1}
$$

On the other hand, if $v \in H(\operatorname{div}, \Omega)$, the trace $v \cdot \nu_{2}=-v \cdot \nu_{1}$ is denoted by $v \cdot \nu$. Hence, the unit normal vector $\nu=\nu_{2}$ has been chosen such that it is pointing into $\Omega_{1}$ (cf. Figure 3.1).

Throughout the paper, the discrete "pressure space" $Q=Q_{h}$ is assumed to be of the form $Q=Q_{1} \oplus Q_{2}$, where $Q_{i} \subset L^{2}\left(\Omega_{i}\right)$. This corresponds to the requirement that the interior boundary $\Gamma$ is a "mesh-line" for the grid generating the space $Q$. Similarly, we assume that $V_{1}=V_{1, h}$ and $V_{2}=V_{2, h}$ are finite element spaces such that

$$
V_{i} \subset H\left(\operatorname{div}, \Omega_{i}\right),
$$

and we let $\widetilde{V} \subset H\left(\operatorname{div}, \Omega_{1}\right) \oplus H\left(\operatorname{div}, \Omega_{2}\right)$ be given by $\widetilde{V}=V_{1} \oplus V_{2}$. We observe that

$$
H(\operatorname{div}, \Omega)=\left\{v \in H\left(\operatorname{div}, \Omega_{1}\right) \oplus H\left(\operatorname{div}, \Omega_{2}\right):[v \cdot \nu]=0\right\} .
$$

The desired finite element space $V$ occurring in the system (2.4) is similarly given by

$$
V=\{v \in \tilde{V}:[v \cdot \nu]=0\},
$$

where obviously $V \subset H(\operatorname{div}, \Omega)$. However, the larger space $\widetilde{V}$ will be used in $\S 6$ in order to construct a preconditioner for (2.4).

We also introduce spaces consisting of the normal components of the traces on $\Gamma$ of functions in $V_{i}$. We let $S_{i}=S_{i, h}(\Gamma)$ be the linear spaces given by $S_{i}=\left\{v \cdot \nu_{i}: v \in V_{i}\right\}$. We will assume throughout this paper that $S_{1}=S_{2}$, and this linear space will be denoted $S=S_{h}(\Gamma)$. We observe that it follows from the continuity property of the map (3.1) that $S \subset H^{-1 / 2}(\Gamma)$. We shall in fact assume that $S \subset L^{2}(\Gamma)$. This will usually always be the case, since in practice $S$ typically consists of discontinuous piecewise polynomials.

In order to describe some of the preconditioners below we will also need a space of continuous functions on $\Gamma$. Associated with the space $S_{h}(\Gamma)$, the existence of finite element subspaces $S^{*}=S_{h}^{*}(\Gamma)$ of $H_{0}^{1}(\Gamma)$ is assumed. If $S$ is a space of piecewise constants, then $S^{*}$ will typically consist of continuous piecewise linear functions. The spaces $S$ and $S^{*}$ are assumed to be related such that for any nonzero element $\chi \in S$

$$
\sup _{\mu \in S^{*}}\langle\mu, \chi\rangle_{\Gamma}>0 \text {. }
$$

We observe that (3.2) in particular implies that $\operatorname{dim}\left(S^{*}\right) \geq \operatorname{dim}(S)$.

The spaces $\widetilde{V}, Q$, and $S^{*}$ will also be assumed to be related such that a proper interpolation operator exists. The desired "inf-sup" condition (2.5) will then be derived from this interpolation property. We assume that there exists a family of interpolation operators $\Pi=\Pi_{h}$ from $H\left(\operatorname{div}, \Omega_{1}\right) \oplus H\left(\operatorname{div}, \Omega_{2}\right)$ into $\widetilde{V}$, bounded uniformly in $h$, such that

(3.3) $\tilde{b}(\Pi v-v, q)+\sum_{i=1}^{2}\left\langle(\Pi v-v) \cdot \nu_{i}, \mu_{i}\right\rangle_{\Gamma}=0 \quad \forall\left(q, \mu_{1}, \mu_{2}\right) \in Q \times S^{*} \times S^{*}$. 
Here, $\tilde{b}$ is the extension of the bilinear form $b$ given by

$$
\tilde{b}(v, q)=-\int_{\Omega_{1}} q \nabla \cdot v d x-\int_{\Omega_{2}} q \nabla \cdot v d x .
$$

We observe that (3.2) and (3.3) imply that if $v \in H(\operatorname{div}, \Omega)$, then $\Pi v \in V$. Furthermore, for any $v \in H(\operatorname{div}, \Omega)$,

$$
b(\Pi v-v, q)=0 \quad \forall q \in Q .
$$

Hence, condition (2.5) follows from the fact that this condition holds with the space $V$ replaced by $H(\operatorname{div}, \Omega)$ (cf. [16]).

We shall also require that $(2.6)$ holds on each subdomain, i.e.,

$$
\sup _{q \in Q} \tilde{b}(v, q)>0
$$

for all $v \in \tilde{V}$ with $\nabla \cdot v \neq 0$.

Finally, we assume that the velocity space $V$ satisfies an "inverse inequality" of the form

$$
|v \cdot \nu|_{\Gamma}^{2} \leq c h^{-1}\|v\|^{2} \quad \forall v \in V .
$$

Such an inequality typically holds if the finite element space $V$ is constructed on a uniform or quasi-uniform grid.

\section{THE PRECONDITIONED ITERATIVE METHOD}

In this section we shall describe the preconditioned minimum residual method for saddle point problems of the form (1.2), and discuss the application of this method to the solution of linear systems of the form (2.4). Throughout this section, $\langle\cdot, \cdot\rangle$ denotes an inner product on $\mathbb{R}^{m}$ and $\mathbb{R}^{n}$, and $|\cdot|$ denotes the corresponding norms.

Recall that the coefficient matrix of the linear system (1.2) is given by

$$
\mathscr{A}=\left(\begin{array}{cc}
A & B \\
B^{T} & 0
\end{array}\right),
$$

where $A \in \mathbb{R}^{m \times m}$ and $B \in \mathbb{R}^{m \times n}$ with $\operatorname{rank}(B)=n$. Furthermore, $A$ is symmetric and positive definite, and $B^{T}$ is the transpose of $B$ with respect to the given inner products.

The minimum residual method is an iterative method for general symmetric, nonsingular systems. Consider a system of the form $\mathscr{A} \alpha^{*}=\beta$, and, for $k \geq 1$, let $V_{k}$ denote the Krylov space

$$
V_{k}=\operatorname{span}\left\{\beta, \mathscr{A} \beta, \ldots, \mathscr{A}^{k-1} \beta\right\} .
$$

The approximation $\alpha_{k} \in V_{k}$ of $\alpha^{*}$, obtained after $k-1$ iterations, is uniquely determined by the residual property

$$
\left|\beta-\mathscr{A} \alpha_{k}\right|^{2}=\inf _{\alpha \in V_{k}}|\beta-\mathscr{A} \alpha|^{2}
$$

We refer to $[24,27]$ for details on the iterative algorithm which generates the vectors $\alpha_{k}$. For the discussion here it is important to recall that typically the coefficient matrix $\mathscr{A}$ has to be multiplied with a vector once for each iteration, and that the algorithm depends on the chosen inner products. 
In [27] we also discuss the convergence properties of the minimum residual method applied to systems of the form (1.2). It is established there that the convergence rate of the method is dominated by three parameters. These are the condition numbers of the matrices $A$ and $B$, and a third parameter measuring the relative scaling between them.

Let $\lambda_{0}$ and $\lambda_{1}$ denote the smallest and largest eigenvalue of $A$, respectively. Similarly, we let $\sigma_{0}$ and $\sigma_{1}$ denote the extreme singular values of the rectangular matrix $B$; i.e., $\sigma_{0}^{2}$ and $\sigma_{1}^{2}$ are the extreme eigenvalues of $B^{T} B$. The spectral condition numbers of the matrices $A$ and $B$ are then given by

$$
\kappa(A)=\lambda_{1} / \lambda_{0} \quad \text { and } \quad \kappa(B)=\sigma_{1} / \sigma_{0} .
$$

Furthermore, the relative scaling parameter, $\rho=\rho(B, A)$, is given by

$$
\rho=\sigma_{0} / \lambda_{0} \text {. }
$$

We observe that the adjoint operation $B \rightarrow B^{T}$ depends on the chosen inner product. Hence, the quantities $\kappa(B)$ and $\rho(B, A)$ are also inner product dependent.

In order to guarantee fast convergence of the method, the scaling parameter $\rho$ should be of order one, i.e., neither too small nor too large. On the other hand, if $\rho$ is kept roughly fixed, the convergence rate will usually increase with decreasing values of $\kappa(A)$ and $\kappa(B)$. Hence, the purpose of a preconditioner for a properly scaled system is to tranform it into a new system of the form (1.2), with $\rho$ essentially unchanged, but where the condition numbers of the matrices $A$ and/or $B$ have been decreased.

The preconditioned minimum residual method discussed in [27] is defined by two symmetric, positive definite matrices $M \in \mathbb{R}^{m \times m}$ and $N \in \mathbb{R}^{n \times n}$. We let $\mathscr{B}$ denote the block diagonal matrix

$$
\mathscr{B}=\left(\begin{array}{cc}
M & 0 \\
0 & N
\end{array}\right)
$$

The new preconditioned system now takes the form

$$
\mathscr{B}^{-1} \mathscr{A}\left(\begin{array}{l}
\xi \\
\eta
\end{array}\right)=\mathscr{B}^{-1}\left(\begin{array}{l}
b \\
c
\end{array}\right)
$$

This system is clearly equivalent to (1.2), and the coefficient matrix, $\mathscr{B}^{-1} \mathscr{A}$, is given by

$$
\mathscr{B}-1 \mathscr{A}=\left(\begin{array}{cc}
M^{-1} A & M^{-1} B \\
N^{-1} B^{T} & 0
\end{array}\right)
$$

Furthermore, the matrix $\mathscr{B}^{-1} \mathscr{A}$ is symmetric in an appropriate inner product. In order to see this, define a new inner product on $\mathbb{R}^{m}$ by $[\xi, \chi]_{M}=\langle M \xi, \chi\rangle$. Similarly, let $[\eta, \theta]_{N}=\langle N \eta, \theta\rangle$ be an inner product on $\mathbb{R}^{n}$, and finally define the inner product $[\cdot, \cdot]$ on $\mathbb{R}^{m} \times \mathbb{R}^{n}$ by

$$
[(\xi, \eta),(\chi, \theta)]=[\xi, \chi]_{M}+[\eta, \theta]_{N} \text {. }
$$

Then $\mathscr{B}^{-1} \mathscr{A}$ is symmetric in the inner product $[\cdot, \cdot]$. Furthermore, $M^{-1} A$ is symmetric and positive definite in the inner product $[\cdot, \cdot]_{M}$, and $N^{-1} B^{T}$ is the adjoint of $M^{-1} B$ with respect to the two new inner products on $\mathbb{R}^{m}$ and $\mathbb{R}^{n}$. The preconditioned system (4.3) has therefore the same saddle point structure as the original system (1.2). Consequently, the minimum residual 
method, with the inner product $\langle\cdot, \cdot\rangle$ replaced by $[\cdot, \cdot]$, can be applied to the new system.

In order to obtain an effective preconditioner, the matrix $\mathscr{B}$ must satisfy two properties. First, it should be easily invertible, or more precisely, it should be easy to solve linear systems with $\mathscr{B}$ as a coefficient matrix. This is because a system of this form has to be solved for each iteration. We observe that, since $\mathscr{B}$ is block diagonal, this is equivalent to require that the two matrices $M$ and $N$ are easily invertible. The second necessary property is that the minimum residual method applied to the preconditioned system should converge rapidly. From the discussion above we recall that, under the assumption of a proper scaling, this is the case if the condition numbers of the matrices $M^{-1} A$ and $M^{-1} B$ are sufficiently small.

We recall that the condition numbers and the scaling parameter are in general dependent on the inner products through the adjoint operation. We let $\hat{\kappa}$ and $\hat{\rho}$ denote these functions with respect to the new inner products $[\cdot, \cdot]$ introduced above.

The condition number of $M^{-1} A, \hat{\kappa}\left(M^{-1} A\right)$, is frequently estimated by establishing inequalities of the form

$$
\hat{\lambda}_{0}|\chi|_{M}^{2} \leq\left[M^{-1} A \chi, \chi\right]_{M} \leq \hat{\lambda}_{1}|\chi|_{M}^{2} \quad \forall \chi \in \mathbb{R}^{m},
$$

for suitable constants $\hat{\lambda}_{0}$ and $\hat{\lambda}_{1}$, since these inequalities imply that

$$
\hat{\kappa}\left(M^{-1} A\right) \leq \hat{\lambda}_{1} / \hat{\lambda}_{0} .
$$

Here, $|\cdot|_{M}$ denotes the norm corresponding to the inner product $[\cdot, \cdot]_{M}$. Furthermore, from the definitions of the inner products we find that these inequalities are equivalent to

$$
\hat{\lambda}_{0}\langle M \chi, \chi\rangle \leq\langle A \chi, \chi\rangle \leq \hat{\lambda}_{1}\langle M \chi, \chi\rangle \quad \forall \chi \in \mathbb{R}^{m} .
$$

Similarly, we derive that $\hat{\kappa}\left(M^{-1} B\right)$ is bounded by $\hat{\sigma}_{1} / \hat{\sigma}_{0}$, where $\hat{\sigma}_{0}$ and $\hat{\sigma}_{1}$ are constants such that

$$
\hat{\sigma}_{0}^{2}\langle N \theta, \theta\rangle \leq\left\langle B^{T} M^{-1} B \theta, \theta\right\rangle \leq \hat{\sigma}_{1}^{2}\langle N \theta, \theta\rangle \quad \forall \theta \in \mathbb{R}^{n} .
$$

The second requirement for $\mathscr{B}$ is therefore fulfilled if $M$ and $N$ are symmetric, positive definite matrices such that (4.5) and (4.6) hold with ratios $\hat{\lambda}_{1} / \hat{\lambda}_{0}$ and $\hat{\sigma}_{1} / \hat{\sigma}_{0}$ sufficiently close to one. Our conclusion is therefore that $\mathscr{B}$ is an effective preconditioner for $\mathscr{A}$ if $M$ and $N$ are effective preconditioners for the symmetric, positive definite matrices $A$ and $B^{T} M^{-1} B$, respectively.

Note that we are not interested in the matrices themselves. However, we must be able to calculate the action of $M^{-1}$ and $N^{-1}$, since this has to be done once in each iteration of the iterative method.

Consider now the saddle point problem (2.4). Recall that the matrix $A$ corresponds to the bilinear form $a(u, v)$, defined in (2.3). It follows directly from (2.1) that

$$
\tau_{0}\|v\|^{2} \leq a(v, v) \leq \tau_{1}\|v\|^{2} \quad \forall v \in V
$$

with constants $\tau_{0}$ and $\tau_{1}$. We note that, if $\langle\cdot, \cdot\rangle$ is the inner product on $\mathbb{R}^{m}$ induced by the $L^{2}$-product on $V$, this corresponds to (4.5) with $M$ equal to the identity matrix and $\hat{\lambda}_{i}=\tau_{i}$. Hence, if the ratio $\tau_{1} / \tau_{0}$ is not too large, the identity is an acceptable preconditioner for $A$. Throughout this paper, we will 
therefore only consider preconditioners where $M=I$. We observe, in particular, that the condition number of $A$ is independent of the mesh parameter $h$.

Having chosen $M=I$, we turn our attention to the problem of constructing a preconditioner for $B^{T} B$. We start by deriving an expression for the bilinear form corresponding to $B^{T} B$. Define the discrete gradient operator $\nabla_{h}: Q \mapsto$ $V$, corresponding to the matrix $B$ in (1.2), by

$$
\left(\nabla_{h} q, v\right)=b(v, q) \quad \forall q \in Q, \forall v \in V .
$$

Since $b(v, q)=-(q, \nabla \cdot v)$, the adjoint of $B$, corresponding to the $L^{2}$ products on $V$ and $Q$, is $-P_{0}(\nabla \cdot)$. Here, $P_{0}$ is an $L^{2}$-projection onto $Q$. The inequality (4.6) therefore takes the form

$$
\hat{\sigma}_{0}^{2} N(q, q) \leq\left(\nabla_{h} q, \nabla_{h} q\right) \leq \hat{\sigma}_{1}^{2} N(q, q) \quad \forall q \in Q,
$$

where $N(\cdot, \cdot)$ is the bilinear form associated with the matrix $N$. Hence, $N$ should be chosen as a preconditioner for the nonconforming discrete Laplace operator $-P_{0} \nabla \cdot \nabla_{h}$.

Consider the discrete Poisson equation of the form

$$
\left(\nabla_{h} r, \nabla_{h} q\right)=(l, q) \quad \forall q \in Q,
$$

where the unknown function $r \in Q$. The condition (2.5) implies that this problem has a unique solution. Furthermore, it is easy to see that (4.10) is equivalent to the following problem of the form (2.4):

$$
\begin{array}{rlrl}
(w, v)+b(v, r) & =0, & & \forall v \in V, \\
b(w, q) & =-(l, q) & \forall q \in Q,
\end{array}
$$

where $w=-\nabla_{h} r$. Hence, the design of effective preconditioners $N$ is closely related to the properties of this saddle point problem.

Finally, we observe that when $M=I$ the proper scaling factor for the preconditioned system, $\hat{\rho}(B, A)$, satisfies the inequality

$$
\hat{\sigma}_{0} / \tau_{1} \leq \hat{\rho}(B, A) \leq \hat{\sigma}_{1} / \tau_{0} .
$$

Hence, since $\tau_{0}$ and $\tau_{1}$ are fixed constants, the properties of a preconditioner are determined by the two constants $\hat{\sigma}_{0}$ and $\hat{\sigma}_{1}$ appearing in (4.9).

\section{The NeumanN PReCONDITIONER}

In this section we shall describe a domain decomposition preconditioner for the linear system (2.4) based on subproblems with Neumann boundary conditions on the interior boundary $\Gamma$. We recall that our purpose is to design a preconditioner $N$ such that the inequality (4.9) holds with the ratio $\hat{\sigma}_{1} / \hat{\sigma}_{0}$ sufficiently close to one. If no preconditioning is performed, the bilinear form $N$ corresponds to the $L^{2}$ inner product on $Q$. In this case, (4.9) holds with $\hat{\sigma}_{1} / \hat{\sigma}_{0}=O\left(h^{-1}\right)$. The preconditioner studied below will reduce this ratio to $O\left(h^{-1 / 2}\right)$. Hence, the bound still grows with the size of the system, but more slowly than with no preconditioner. These bounds therefore indicate that the preconditioner will speed up the convergence of the iterative method considerably, but that the number of iterations required by the preconditioned minimum residual method will still increase with the dimension of the system. The numerical experiments, which will be presented in $\S 7$, will indeed confirm these expectations. 
We introduce the subspace $H_{0}(\operatorname{div}, \Omega)$ of $H(\operatorname{div}, \Omega)$ given by

$$
H_{0}(\operatorname{div}, \Omega)=\{v \in H(\operatorname{div}, \Omega): v \cdot \nu=0\} \text {. }
$$

Similarly, we let $V_{0} \subset V$ be given by $V_{0}=\{v \in V: v \cdot \nu=0\}$. Note that it follows from (3.2) and (3.3) that the interpolation operator $\Pi$ maps $H_{0}(\operatorname{div}, \Omega)$ into $V_{0}$. Furthermore, $\Pi v$ satisfies, for any $v \in H_{0}(\operatorname{div}, \Omega)$,

$$
b(\Pi v-v, q)=0 \quad \forall q \in Q .
$$

Hence, as above, the discrete inf-sup condition

$$
\inf _{q \in Q} \sup _{v \in V_{0}} \frac{b(v, q)}{\|q\|\|v\|_{\text {div }}} \geq \gamma_{0}>0
$$

follows from the corresponding continuous condition with $V_{0}$ replaced by $H_{0}(\operatorname{div}, \Omega)$. This latter condition is again equivalent to inf-sup conditions on the two subdomains.

Let $\chi \in S$ be given and assume that $\varphi$ solves the boundary value problem

$$
\begin{aligned}
\Delta \varphi=0 & \text { in } \Omega_{1} \cup \Omega_{2}, \\
\varphi=0 & \text { on } \partial \Omega, \\
\nabla \varphi \cdot \nu=\chi & \text { on } \Gamma .
\end{aligned}
$$

Here, $\Delta$ denotes the Laplace operator. We observe that the uniquely determined function $\varphi$ is a harmonic function on each subdomain. Let $\psi=-\nabla \varphi$. Since $\nabla \cdot \psi=0$ on each subdomain, it follows from elliptic theory (cf., e.g., [25]) that $\psi$ and $\varphi$ satisfy the a priori estimate

$$
\|\psi\|_{\text {div }}+\|\varphi\| \leq c|\chi|_{-1 / 2, \Gamma}
$$

Define $w \in V$ by $w=\Pi \psi$. From the continuity property of the interpolation operator $\Pi$ we then obtain $\|w\|_{\text {div }} \leq c|\chi|_{-1 / 2, \Gamma}$. The following result has therefore been established.

Lemma 5.1. There is a constant $c$, independent of $h$, such that for any $\chi \in S$

$$
\inf \left\{\|w\|_{\text {div }}: w \in V, w \cdot \nu=\chi\right\} \leq c|\chi|_{-1 / 2, \Gamma} .
$$

Define a new discrete gradient operator, $\nabla_{h}^{0}: Q \mapsto V_{0}$, by

$$
\left(\nabla_{h}^{0} q, v\right)=b(v, q) \quad \forall q \in Q, \forall v \in V_{0} .
$$

The bilinear form $N_{0}$ which defines the Neumann preconditioner is now given by $N_{0}(r, q)=\left(\nabla_{h}^{0} r, \nabla_{h}^{0} q\right)$. This bilinear form is obviously symmetric, and (5.1) implies that

$$
\left\|\nabla_{h}^{0} q\right\|=\sup _{v \in V_{0}} \frac{\left(\nabla_{h}^{0} q, v\right)}{\|v\|} \geq \sup _{v \in V_{0}} \frac{b(v, q)}{\|v\|_{\text {div }}} \geq \gamma_{0}\|q\| .
$$

Hence,

$$
N_{0}(q, q)=\left\|\nabla_{h}^{0} q\right\|^{2} \geq \gamma_{0}^{2}\|q\|^{2}
$$

for all $q \in Q$. Consider the problem

$$
N_{0}(r, q)=(l, q) \quad \forall q \in Q
$$


where the unknown function $r \in Q=Q_{1} \oplus Q_{2}$. If we let $w=-\nabla_{h}^{0} r$, then $(w, r) \in V_{0} \times Q$ is the solution of the saddle point problem

$$
\begin{aligned}
(w, v)+b(v, r) & =0, & & \forall v \in V_{0}, \\
b(w, q) & =-(l, q) & & \forall q \in Q .
\end{aligned}
$$

It is easy to see that this problem decouples into saddle point problems on each subdomain. Hence, with the proper choice of finite element spaces, this problem can be solved by a fast solver (cf. $\S 7$ ).

The purpose of the rest of this section is therefore to discuss the efficiency of this preconditioner, i.e., to derive an inequality of the form (4.9).

For each $q \in Q$, let $\nabla_{h}^{\Gamma} q \in V$ be given by

$$
\nabla_{h}^{\Gamma} q=\nabla_{h} q-\nabla_{h}^{0} q .
$$

Note that it follows from (4.8) and (5.4) that $\left(\nabla_{h}^{\Gamma} q, v\right)=0$ for all $v \in V_{0}$. In particular, $\left(\nabla_{h}^{\Gamma} q, \nabla_{h}^{0} q\right)=0$, and hence

$$
\left\|\nabla_{h} q\right\|^{2}=\left\|\nabla_{h}^{0} q\right\|^{2}+\left\|\nabla_{h}^{\Gamma} q\right\|^{2}
$$

It therefore follows that the left side of inequality (4.9) is satisfied with $\hat{\sigma}_{0}=1$. The following result shows that $\hat{\sigma}_{1}=O\left(h^{-1 / 2}\right)$.

Theorem 5.2. There is a constant $c$, independent of $h$, such that

$$
\left\|\nabla_{h} q\right\| \leq c h^{-1 / 2}\left\|\nabla_{h}^{0} q\right\| \quad \forall q \in Q .
$$

Proof. Introduce the bilinear form $R(v, q)$ given by

$$
R(v, q)=b(v, q)-\left(\nabla_{h}^{0} q, v\right) .
$$

By (4.8) and (5.4), it follows that

$$
\left(\nabla_{h}^{\Gamma} q, v\right)=R(v, q) \quad \forall v \in V .
$$

Furthermore, $R(v, q)=0$ for all $v \in V_{0}$. Therefore, for each given $q \in Q$, the bilinear form $R(\cdot, q)$ can be considered to be a linear functional on $S=S_{h}(\Gamma)$. Hence, for each $q \in Q$ there exists a unique element $[q] \in S$ such that

$$
\langle[q], v \cdot \nu\rangle_{\Gamma}=R(v, q) \quad \forall v \in V .
$$

The element $[q] \in S$ should be interpreted as the "jump of $q$ at $\Gamma$." The relation (5.8) can now be rewritten in the form

$$
\left(\nabla_{h}^{\Gamma} q, v\right)=\langle[q], v \cdot \nu\rangle \quad \forall v \in V .
$$

Hence, by the inverse assumption (3.5),

$$
\begin{aligned}
\left\|\nabla_{h}^{\Gamma} q\right\| & =\sup _{v \in V} \frac{\left(\nabla_{h}^{\Gamma} q, v\right)}{\|v\|}=\sup _{v \in V} \frac{\langle[q], v \cdot \nu\rangle_{\Gamma}}{\|v\|} \\
& \leq|[q]|_{\Gamma} \sup _{v \in V} \frac{|v \cdot \nu|_{\Gamma}}{\|v\|} \leq c h^{-1 / 2}|[q]|_{\Gamma}
\end{aligned}
$$

or

$$
\left\|\nabla_{h}^{\Gamma} q\right\| \leq c h^{-1 / 2}|[q]|_{\Gamma},
$$

where the constant $c$ in independent of $q$ and $h$. 
On the other hand, from (5.9) we obtain

$$
\begin{aligned}
|[q]|_{\Gamma} & =\sup _{\chi \in S} \frac{\langle[q], \chi\rangle_{\Gamma}}{|\chi|_{\Gamma}}=\sup _{\chi \in S} \frac{R(w, q)}{\|w\|_{\text {div }}} \frac{\|w\|_{\text {div }}}{|\chi|_{\Gamma}} \\
& \leq\left(\|q\|^{2}+\left\|\nabla_{h}^{0} q\right\|^{2}\right)^{1 / 2} \sup _{\chi \in S} \frac{\|w\|_{\text {div }}}{|\chi|_{\Gamma}}
\end{aligned}
$$

where $w \in V$ is any function such that $w \cdot \nu=\chi$ on $\Gamma$. Hence, from (5.5) and by selecting $w$ such that $\|w\|_{\text {div }} /|\chi|_{-1 / 2, \Gamma}$ is minimal, we obtain from Lemma 5.1 that $|[q]|_{\Gamma} \leq c\left\|\nabla_{h}^{0} q\right\|$. Together with (5.10) this implies that there is a constant $c$, independent of $q$ and $h$, such that

$$
\left\|\nabla_{h}^{\Gamma} q\right\| \leq c h^{-1 / 2}\left\|\nabla_{h}^{0} q\right\| \text {. }
$$

We therefore conclude that

$$
\left\|\nabla_{h} q\right\|=\left(\left\|\nabla_{h}^{0} q\right\|^{2}+\left\|\nabla_{h}^{\Gamma} q\right\|^{2}\right)^{1 / 2} \leq c h^{-1 / 2}\left\|\nabla_{h}^{0} q\right\|
$$

for a suitable constant $c$.

\section{A DiRICHLET PRECONDITIONER}

The domain decomposition preconditioner developed in the previous section was based on subproblems with a Neumann boundary condition on the interior boundary $\Gamma$. In contrast, the efficient domain decomposition preconditioners for systems arising from conforming finite element discretizations of elliptic equations are based on the solution of some subproblems with an interior Dirichlet condition (cf., e.g., [3, 6] or [7]). In this section we shall develop corresponding preconditioners for the systems arising from mixed finite element methods. The preconditioners studied below are based on the solution of subproblems with Dirichlet boundary conditions on the interior boundary, and correspond to the ones studied in $[3,6]$. Since these preconditioners involve independent problems on each subdomain, they can, as in [6], be generalized to domains with a more complex substructure. However, in this paper we will only consider the two subdomains case studied above. In particular, we will show that, in the case of two subdomains, the appropriate condition numbers are independent of the discretization parameter $h$.

Since the Dirichlet boundary conditions are natural boundary conditions in the mixed finite element method, the discrete system $(2.4)$ will be slightly generalized. Instead of (2.4) we introduce the following generalized system:

Find $\left(u_{h}, p_{h}, \lambda_{h}\right) \in \widetilde{V} \times Q \times S^{*}$ such that

$$
\begin{aligned}
a\left(u_{h}, v\right)+\tilde{b}\left(v, p_{h}\right)+\left\langle\lambda_{h},[v \cdot \nu]\right\rangle_{\Gamma} & =G(v) & & \forall v \in \tilde{V}, \\
\tilde{b}\left(u_{h}, q\right) & =F(q) & & \forall q \in Q, \\
\left\langle\mu,\left[u_{h} \cdot \nu\right]\right\rangle_{\Gamma} & =0 & & \forall \mu \in S^{*},
\end{aligned}
$$

where we recall that the space $\widetilde{V}$ and the bilinear form $\tilde{b}(\cdot, \cdot)$ are defined in $\S 3$. In particular, the extended form $\tilde{b}$ ignores the possible jumps at $\Gamma$. A similar convention is used for the norm $\|\cdot\|_{\text {div }}$ below, i.e., the norm is defined by summing the contributions from each subdomain.

The system (6.1) arises naturally if the elliptic equation (1.1) is discretized by a mixed finite element method on each subdomain, and if the interior boundary 
conditions $[p]=0$ and $[u \cdot \nu]=0$ are required on $\Gamma$. We observe that $(6.1)$ is a generalization of (2.4) in the sense that if the triple $\left(u_{h}, p_{h}, \lambda_{h}\right)$ solves (6.1) then the pair $\left(u_{h}, p_{h}\right)$ solves (2.4). Furthermore, $\lambda_{h} \in S^{*}$ is an approximation of the trace of $p$ on $\Gamma$.

The system (6.1) has the saddle point structure (1.2). The following consequence of the assumptions given above shows that this system satisfies the proper inf-sup condition.

Lemma 6.1. There exists a constant $\gamma_{1}$, independent of the mesh parameter $h$, such that

$$
\inf _{(q, \mu) \in Q \times S^{*}} \sup _{v \in \widetilde{V}} \frac{\tilde{b}(v, q)+\langle\mu,[v \cdot \nu]\rangle_{\Gamma}}{\left(\|q\|+|\mu|_{1 / 2, \Gamma)}\right)\|v\|_{\text {div }}} \geq \gamma_{1}>0 .
$$

Proof. As above, the result follows from a proper application of the interpolation property (3.3). Let $(q, \mu) \in Q \times S^{*}$ be given, and consider elliptic equations of the form

$$
\begin{aligned}
\Delta \varphi=q & \text { in } \Omega_{1} \cup \Omega_{2}, \\
\varphi=0 & \text { on } \partial \Omega, \\
{[\varphi]=0 } & \text { on } \Gamma, \\
{[\nabla \varphi \cdot \nu]=\chi } & \text { on } \Gamma,
\end{aligned}
$$

where $\chi \in H^{-1 / 2}(\Gamma)$ is arbitrary. Let $\psi=\nabla \varphi$. Then $\psi \in H\left(\operatorname{div}, \Omega_{1}\right) \oplus$ $H\left(\operatorname{div}, \Omega_{2}\right)$ and from elliptic regularity (cf. [18]) it follows that

$$
\|\psi\|_{\text {div }}+\|\varphi\| \leq c\left(\|q\|+|\chi|_{-1 / 2, \Gamma}\right)
$$

for a suitable constant $c$ independent of $q$ and $\chi$. Furthermore,

$$
\tilde{b}(\psi, q)+\langle\mu,[\psi \cdot \nu]\rangle_{\Gamma}=\|q\|^{2}+\langle\mu, \chi\rangle_{\Gamma} .
$$

By choosing $\chi$ such that $|\chi|_{-1 / 2, \Gamma}=|\mu|_{1 / 2, \Gamma}$ and $\langle\mu, \chi\rangle_{\Gamma} \geq|\mu|_{1 / 2, \Gamma}^{2} / 2$, we therefore obtain from (6.4) that

$$
\frac{\tilde{b}(\psi, q)+\langle\mu,[\psi \cdot \nu]\rangle}{\|\psi\|_{\text {div }}} \geq c\left(\|q\|+|\mu|_{1 / 2, \Gamma}\right)
$$

for a suitable positive constant $c$.

Let $v \in \widetilde{V}$ be given by $v=\Pi \psi$. Since $\|v\|_{\text {div }} \leq c\|\psi\|_{\text {div }}$ and (3.3) implies that

$$
\tilde{b}(\psi, q)+\langle\mu,[\psi \cdot \nu]\rangle_{\Gamma}=\tilde{b}(v, q)+\langle\mu,[v \cdot \nu]\rangle_{\Gamma},
$$

the desired result now follows from (6.5).

Define the extended discrete gradient operator $\tilde{\nabla}_{h}: Q \times S^{*} \mapsto \widetilde{V}$ by

$$
\left(\tilde{\nabla}_{h}(q, \mu), v\right)=\tilde{b}(v, q)+\langle\mu,[v \cdot \nu]\rangle_{\Gamma} \quad \forall(q, \mu) \in Q \times S^{*}, \forall v \in \widetilde{V} .
$$

This extended gradient operator is associated with the system (6.1) in the same way as the operator $\nabla_{h}$ is associated with (2.4). Therefore, in order to design an effective preconditioner for this system, the bilinear form

$$
\left(\widetilde{\nabla}_{h}(r, \eta), \widetilde{\nabla}_{h}(q, \mu)\right) \text {, }
$$

defined on $\left(Q \times S^{*}\right)^{2}$, has to be preconditioned. 
We note that, for any $(r, \eta) \in Q \times S^{*}$,

$$
\begin{aligned}
\left\|\widetilde{\nabla}_{h}(r, \eta)\right\| & =\sup _{v \in \widetilde{V}} \frac{\left(\widetilde{\nabla}_{h}(r, \eta), v\right)}{\|v\|} \\
& \geq \sup _{v \in \widetilde{V}} \frac{\left(\widetilde{\nabla}_{h}(r, \eta), v\right)}{\|v\|_{\text {div }}}=\sup _{v \in \widetilde{V}} \frac{\tilde{b}(v, r)+\langle\eta,[v \cdot \nu]\rangle_{\Gamma}}{\|v\|_{\text {div }}} .
\end{aligned}
$$

Hence, we derive from (6.2) that

$$
\left\|\widetilde{\nabla}_{h}(r, \eta)\right\| \geq \gamma_{1}\left(\|r\|+|\eta|_{1 / 2, \Gamma}\right) .
$$

The bilinear form (6.7) is therefore an inner product on $Q \times S^{*}$.

In order to define the desired preconditioners, we consider an orthogonal projection of elements in the product space $Q \times S^{*}$ into $Q \times\{0\}$ with respect to the inner product (6.7). For an arbitrary element $(r, \eta) \in Q \in S^{*}$ consider the unique orthogonal decomposition of the form

$$
(r, \eta)=\left(r^{0}, 0\right)+\left(r^{H}, \eta\right) .
$$

Hence, $r^{0} \in Q$ is determined by

$$
\left(\widetilde{\nabla}_{h}\left(r^{0}, 0\right), \widetilde{\nabla}_{h}(q, 0)\right)=\left(\widetilde{\nabla}_{h}(r, \eta), \widetilde{\nabla}_{h}(q, 0)\right) \quad \forall q \in Q .
$$

Furthermore, if we let $w^{0}=-\widetilde{\nabla}_{h}\left(r^{0}, 0\right)$, then the pair $\left(w^{0}, r^{0}\right) \in \widetilde{V} \times Q$ satisfies the saddle point problem

$$
\begin{aligned}
\left(w^{0}, v\right)+\tilde{b}\left(v, r^{0}\right) & =0 & & \forall v \in \tilde{V}, \\
\tilde{b}\left(w^{0}, q\right) & =-\left(\widetilde{\nabla}_{h}(r, \eta), \widetilde{\nabla}_{h}(q, 0)\right) & & \forall q \in Q .
\end{aligned}
$$

Similarly, if we let $w^{H}=-\widetilde{\nabla}_{h}\left(r^{H}, \eta\right)$, then the triple $\left(w^{H}, r^{H}, \eta\right) \in \widetilde{V} \times Q \times S^{*}$ satisfies

$$
\begin{aligned}
\left(w^{H}, v\right)+\tilde{b}\left(v, r^{H}\right) & =-\langle\eta,[v \cdot \nu]\rangle_{\Gamma} & & \forall v \in \tilde{V}, \\
\tilde{b}\left(w^{H}, q\right) & =0 & & \forall q \in Q .
\end{aligned}
$$

The function $r^{H}$ given in the decomposition above is a mixed finite element approximation of a harmonic extension of the boundary function $\eta$. This observation motivates the following simple result.

Lemma 6.2. Consider the orthogonal decomposition (6.9). There is a constant $c$, independent of $h$, such that

$$
\left\|\widetilde{\nabla}_{h}\left(r^{H}, \eta\right)\right\| \leq c|\eta|_{1 / 2, \Gamma} .
$$

Proof. Since $\tilde{b}\left(w^{H}, r^{H}\right)=0$, it follows immediately from the system (6.12) that

$$
\left\|w^{H}\right\|^{2}=-\left\langle\eta,\left[w^{H} \cdot \nu\right]\right\rangle_{\Gamma} .
$$

Furthermore, since (3.4) implies that $\left\|w^{H}\right\|_{\text {div }}=\left\|w^{H}\right\|$, we obtain that

$$
\left|\left[w^{H} \cdot \nu\right]\right|_{-1 / 2, \Gamma} \leq c\left\|w^{H}\right\|_{\text {div }}=c\left\|w^{H}\right\| .
$$

Hence, the desired result follows from (6.13).

We note that the estimates given by (6.8) and Lemma $6.2 \mathrm{imply}$, in particular, that for any $(r, \eta) \in Q \times S^{*}$

$$
\gamma_{1}|\eta|_{1 / 2, \Gamma} \leq\left\|\widetilde{\nabla}_{h}\left(r^{H}, \eta\right)\right\| \leq c|\eta|_{1,2 / \Gamma} .
$$


Since the decomposition (6.9) is orthogonal, it therefore follows that the two norms

$$
\left\|\widetilde{\nabla}_{h}(r, \eta)\right\| \text { and }\left\|\widetilde{\nabla}_{h}\left(r^{0}, 0\right)\right\|+|\eta|_{1 / 2, \Gamma}
$$

are equivalent, uniformly in $h$, on the space $Q \times S^{*}$.

Motivated by this equivalence, we therefore consider preconditioners of the form

$$
\tilde{N}((r, \eta),(q, \mu))=\left(\widetilde{\nabla}_{h}\left(r^{0}, 0\right), \widetilde{\nabla}_{h}\left(q^{0}, 0\right)\right)+\langle\Lambda \eta, \mu\rangle_{\Gamma}
$$

for the bilinear form (6.7), where $\Lambda: S^{*} \mapsto S^{*}$ is a discrete operator on the interior boundary $\Gamma$ which is symmetric with respect to the inner product $\langle\cdot, \cdot\rangle_{\Gamma}$. The following result is now an obvious consequence of the equivalence (6.14).

Theorem 6.3. Assume that there is a constant $c_{1}$, independent of $h$, such that the operator $\Lambda$ satisfies

$$
c_{1}^{-1}|\eta|_{1 / 2, \Gamma}^{2} \leq\langle\Lambda \eta, \eta\rangle_{\Gamma} \leq c_{1}|\eta|_{1 / 2, \Gamma}^{2} \quad \forall \eta \in S^{*} .
$$

Then there is a constant $c_{2}$, independent of $h$, such that

$$
c_{2}^{-1} \tilde{N}((r, \eta),(r, \eta)) \leq\left\|\widetilde{\nabla}_{h}(r, \eta)\right\|^{2} \leq c_{2} \tilde{N}((r, \eta),(r, \eta)) \quad \forall(r, \eta) \in Q \times S^{*} .
$$

This result shows that if the boundary operator $\Lambda$ is chosen such that $(6.15)$ holds, then the preconditioner $\tilde{N}$ transforms (6.1) into a saddle point problem with conditioning independent of the discretization parameter $h$.

Consider the linear systems of the form

$$
\tilde{N}((r, \eta),(q, \mu))=(l, q)+\left\langle l_{0}, \mu\right\rangle_{\Gamma} \quad \forall(q, \mu) \in Q \times S^{*},
$$

where the data $\left(l, l_{0}\right) \in Q \times S^{*}$ is given. We observe that when $\tilde{N}$ is used as a preconditioner, such a linear system has to be solved once for each iteration. If the operator $\Lambda$ satisfies (6.15) above, then $\tilde{N}$ is positive definite on $Q \times S^{*}$, and hence (6.16) has a unique solution $(r, \eta) \in Q \times S^{*}$. By decomposing this solution according to (6.9), we derive from (6.11) that

$$
\begin{array}{rlrl}
\left(w^{0}, v\right)+\tilde{b}\left(v, r^{0}\right) & =0 & & \forall v \in \tilde{V}, \\
\tilde{b}\left(w^{0}, q\right) & =-(l, q) & \forall q \in Q,
\end{array}
$$

where, as above, $w^{0}=-\widetilde{\nabla}_{h}\left(r^{0}, 0\right) \in \widetilde{V}$. Furthermore, this problem decouples into discrete Poisson equations on each subdomain. With the proper choice of subspaces, this problem can therefore be solved by a fast solver.

When $\left(r^{0}, w^{0}\right) \in Q \times \widetilde{V}$ is computed, it remains to find the orthogonal component of the solution, $\left(r^{H}, \eta\right) \in Q \times S^{*}$. However, it is enough to find $\eta$, since $r^{H}$ can then be calculated from (6.12). This system again corresponds to discrete Poisson equations on each subdomain.

In order to derive a suitable equation for $\eta$, we consider (6.16) with test functions of the form $(q, \mu)=\left(q^{H}, \mu\right)$. Let $w^{H}=-\widetilde{\nabla}_{h}\left(r^{H}, \eta\right)$. Since $\left(w^{H}, w^{0}\right)=0$, we obtain from $(6.17)$ that

$$
\begin{aligned}
\langle\Lambda \eta, \mu\rangle_{\Gamma} & =\left(l, q^{H}\right)+\left\langle l_{0}, \mu\right\rangle_{\Gamma} \\
& =-\tilde{b}\left(w^{0}, q^{H}\right)+\left\langle l_{0}, \mu\right\rangle_{\Gamma}=\left\langle\mu,\left[w^{0} \cdot \nu\right]\right\rangle_{\Gamma}+\left\langle l_{0}, \mu\right\rangle_{\Gamma},
\end{aligned}
$$

or

$$
\Lambda \eta=P^{*}\left[w^{0} \cdot \nu\right]+l_{0}
$$


where $P^{*}: L^{2}(\Gamma) \mapsto S^{*}$ is the $L^{2}$-projection onto $S^{*}$. This equation is a discrete system related to the interior boundary $\Gamma$. Since $\Gamma$ is one-dimensional, we can usually afford to solve such systems (cf. §7).

The results above can be used in two different ways to define preconditioned iterative methods for the system (2.4). From the discussion above, the obvious approach seems to be to replace the system (2.4) by the generalized system (6.1) and then use the bilinear form $\tilde{N}$ as a preconditioner for the form (6.7). However, this strategy leads to approximate solutions of (6.1) (or (2.4)) with a possible small jump on the interior boundary. In the calculations below we have therefore used an alternative approach.

Consider the original discrete system (2.4). Our purpose is to use the equivalence given in Theorem 6.3 only to design a preconditioner for the bilinear form $\left(\nabla_{h} r, \nabla_{h} q\right)$ on $Q^{2}$. In order to see how this can be done, we first observe that if $\widetilde{\nabla}_{h}(r, \eta) \in V$, then $\widetilde{\nabla}_{h}(r, \eta)=\nabla_{h} r$. Furthermore, from (3.2) and (6.2) it follows that for a given $r \in Q$ there is a unique element $\eta(r) \in S^{*}$ such that

$$
\langle\eta,[v \cdot \nu]\rangle_{\Gamma}=\left(\nabla_{h} r, v\right)-\tilde{b}(v, r) \quad \forall v \in \tilde{V},
$$

or equivalently, for each $r \in Q$ there is a unique element $\eta(r) \in S^{*}$ such that $\widetilde{\nabla}_{h}(r, \eta)=\nabla_{h} r$.

Define now the bilinear form $N$ on $Q^{2}$ by

$$
N(r, q)=\tilde{N}((r, \eta(r)),(q, \eta(q))) .
$$

From the discussion above it follows that if the hypothesis of Theorem 6.3 is satisfied, then $N(r, r)$ and $\left(\nabla_{h} r, \nabla_{h} r\right)$ are uniformly equivalent on $Q$. Therefore, the bilinear form $N$ can be used as a preconditioner for the original system (2.4). Furthermore, the linear systems

$$
N(r, q)=(l, q) \quad \forall q \in Q,
$$

which have to be solved for each iteration of the minimum residual method, are equivalent to the system (6.16) with $l_{0}=0$.

The conclusion of this section is that we have generalized the domain decomposition preconditioners studied in $[3,6]$ to the systems derived from the mixed finite element method. In the same way as with conforming finite element methods, these preconditioners require that suitable discrete Poisson equations, together with a system on the interior boundary, can be solved by sufficiently fast solvers.

\section{NUMERICAL EXAMPLES}

The purpose of this section is to present some numerical examples obtained by using the preconditioners developed above. Therefore, in particular, we have to construct spaces $Q, \widetilde{V}, S$, and $S^{*}$ satisfying the desired properties required in $\S 3$.

In the examples below, $\Omega$ will be an L-shaped domain like the one given in Figure 3.1. It is composed of three of the four subsquares obtained by dividing the unit square into four equal squares. The domain is subdivided, in a uniform manner, into axiparallel squares of size $h$. In particular, the grid is chosen such that the interior boundary $\Gamma$ coincides with boundaries of the 


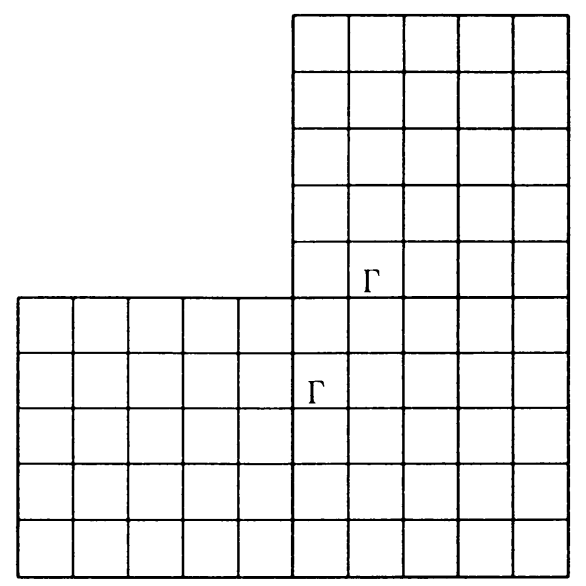

FIGURE 7.1. The domain $\Omega$ with a square grid

squares of the grid (cf. Figure 7.1). On this grid we will apply the quadrilateral Raviart-Thomas elements of order zero (cf. [25]). Hence, the functions in $Q$ are constants on each grid element and, as required in $\S 3, Q=Q_{1} \oplus Q_{2}$, where $Q_{i} \subset$ $L^{2}\left(\Omega_{i}\right)$. Similarly, the components of a vector $v \in \widetilde{V}$ are either constant or linear on each element, and $\widetilde{V}=V_{1} \oplus V_{2}$, with $V_{i} \subset H\left(\operatorname{div}, \Omega_{i}\right)$. Furthermore, the functions in $S$ are piecewise constants on the interior boundary $\Gamma$.

When the above subspaces are applied, the linear systems associated with the subdomains may be solved by fast Poisson solvers. In particular, if a certain combination of the midpoint and the trapezoid rules for numerical integration is used in the evaluation of the integrals, discrete Poisson problems corresponding to the five-point finite difference scheme have to be solved on the subdomains (cf. [26]).

By a proper parametrization each segment of $\Gamma$ can be considered to be the unit interval $I$. The elements of $S$ are then constants on the subintervals of the form $((i-1) \tilde{h}, i \tilde{h})$ for $i=1,2, \ldots, k$, where $k$ is the dimension of $S$ and $\tilde{h}$ is a proper scaling of the grid parameter $h$. On $I$, the space $S^{*}$ is required to be a subspace of $H_{0}^{1}(I)$. We let $S^{*}$ consist of all piecewise linear functions in $H_{0}^{1}(I)$ with possible discontinuities of the slopes at the points $(i-1 / 2) \tilde{h}$ for $i=1,2, \ldots, k$. Hence, $S$ and $S^{*}$ are spaces consisting of functions on $I$ which are piecewise constant or piecewise linear, respectively. However, the piecewise polynomial spaces are generated from different sets of knots. We also observe that

$$
\operatorname{dim}\left(S^{*}\right)=\operatorname{dim}(S)=k .
$$

Consider the assumptions on the spaces $\widetilde{V}, Q, S$, and $S^{*}$ given in $\S 3$. It is easy to see that the spaces $S$ and $S^{*}$ defined on $I$ above satisfy the condition (3.2). In fact, if $\chi \in S$ is given and $\mu \in S^{*}$ interpolates $\chi$ at the points $\theta_{i}$, then

$$
\langle\chi, \mu\rangle_{I} \geq \frac{1}{2}|\chi|_{I}^{2} .
$$

The conditions (3.4) and (3.5) are also easy to check. In particular, (3.4) follows since $\nabla \cdot v \in Q$ for any $v \in \widetilde{V}$, and the inverse assumption (3.5) follows from the uniformity of the grid. 


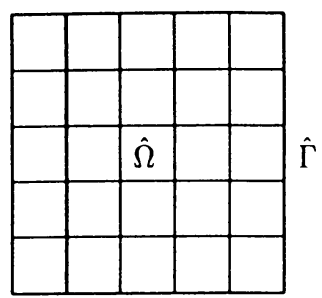

FIGURE 7.2. Reference domain $\widehat{\Omega}$

Finally, in order to verify all the assumptions given in $\S 3$, we have to construct the uniformly bounded interpolation operators

$$
\Pi: H\left(\operatorname{div}, \Omega_{1}\right) \oplus H\left(\operatorname{div}, \Omega_{2}\right) \mapsto \hat{V}
$$

such that the condition (3.3) holds. If the term on the interior boundary had not appeared in (3.3), the construction of a suitable operator $\Pi$ is, e.g., described in [16]. However, in order to extend this construction to the present case, the boundary term has to be treated properly by the operator $\Pi$.

The operator $\Pi$ can be constructed independently on each subdomain. It is therefore sufficient to consider the construction of the interpolation operator on the unit square, $\widehat{\Omega}$, with a regular square grid, and where the interior boundary is represented by the edge $\widehat{\Gamma}=\{(1, y): y \in I\}$ (cf. Figure 7.2).

Furthermore, we let $\widehat{V}$ and $\widehat{Q}$ be the corresponding Raviart-Thomas elements of order zero on $\widehat{\Omega}$. The boundary of $\widehat{\Omega}$ is denoted by $\partial \widehat{\Omega}$, while the exterior unit vector normal to $\partial \widehat{\Omega}$ is $\hat{\nu}$. In order to simplify the notation, we let $S$ and $S^{*}$ denote the finite element spaces on $\widehat{\Gamma}$ implied by the construction above. Below we shall construct interpolation operators $\widehat{\Pi}: H(\operatorname{div}, \widehat{\Omega}) \mapsto \widehat{V}$, uniformly bounded in $h$, such that

$$
\hat{b}(\widehat{\Pi} v-v, q)+\langle\mu,(\widehat{\Pi} v-v) \cdot \hat{\nu}\rangle_{\widehat{\Gamma}}=0 \quad \forall(q, \mu) \in \widehat{Q} \times S^{*},
$$

where $\hat{b}$ is the bilinear form $b$ restricted to $\widehat{\Omega}$. This construction can easily be modified to cover all the three components of the subdomains in the present case. The general condition (3.3) will therefore follow from (7.1).

Since the grid on $\widehat{\Omega}$, in particular, generates a partition of the boundary $\partial \widehat{\Omega}$, the spaces $S$ and $S^{*}$ can be extended, in an obvious way, to discontinuous constants and continuous piecewise linear subspaces of $H^{1}(\partial \widehat{\Omega})$ and $L^{2}(\partial \widehat{\Omega})$, respectively. These spaces on $\partial \widehat{\Omega}$ will be denoted $S_{e}$ and $S_{e}^{*}$. In particular, since the knots of the partition generating the finite element space $S_{e}^{*}$ are located in the middle of the grid edges on $\partial \widehat{\Omega}$, the tangential derivatives of an element in $S_{e}^{*}$ are assumed to be continuous at the corners of $\widehat{\Omega}$. Furthermore, any element $\mu \in S^{*}$ can be extended to an element $\mu_{e}$ of $S_{e}^{*}$ by defining $\mu_{e}$ to be 0 outside $\Gamma$.

It is easy to see that $\operatorname{dim}\left(S_{e}\right)=\operatorname{dim}\left(S_{e}^{*}\right)$, since the degree of freedom for both spaces is equal to the number of subintervals on $\partial \widehat{\Omega}$ generated by the interior grid. Furthermore, by using interpolation relations between the spaces $S_{e}$ and $S_{e}^{*}$ at the midpoints of these subintervals, it is easy to verify that there 
exists a constant $\alpha_{0}>0$, independent of $h$, such that

$$
\inf _{\chi \in S_{e}} \sup _{\mu \in S_{e}^{*}} \frac{\langle\mu, \chi\rangle_{\partial \widehat{\Omega}}}{|\mu|_{\partial \widehat{\Omega}}|\chi|_{\partial \widehat{\Omega}}} \geq \alpha_{0} \text { and } \inf _{\mu \in S_{e}^{*}} \sup _{\chi \in S_{e}} \frac{\langle\mu, \chi\rangle_{\partial \widehat{\Omega}}}{|\mu|_{\partial \widehat{\Omega}}|\chi|_{\partial \widehat{\Omega}}} \geq \alpha_{0} .
$$

In addition to the spaces introduced so far, we will also, for technical reasons, introduce the subspace $Z$ of $H^{1}(\widehat{\Omega})$ consisting of bilinear continuous functions with respect to the grid on $\widehat{\Omega}$. Furthermore, we let $Z_{B}$ denote the restrictions of functions in $Z$ to $\partial \widehat{\Omega}$.

We observe that the space $Z_{B}$ consists of continuous piecewise linear functions on $\partial \widehat{\Omega}$ with respect to the same partition that generates the piecewise constant space $S_{e}$. Hence, translation by half the grid size represents an isomorphism between $Z_{B}$ and $S^{*}$. Also observe that if $\eta \in Z_{B}$, then $\eta_{t} \in S_{e}$, where $\eta_{t}$ is the tangential derivative of $\eta$ in a counterclockwise direction. Furthermore, if $z \in Z$ and $v$ is the divergence-free vector $\left(z_{y},-z_{x}\right)^{T}$, then $v \in \widehat{V}$.

We start the construction of the interpolation operator $\widehat{\Pi}: H(\operatorname{div}, \widehat{\Omega}) \mapsto \widehat{V}$ by introducing two discrete operators on the boundary $\partial \widehat{\Omega}$.

Define $J$ and $J^{*}$, from $L^{2}(\partial \widehat{\Omega})$ into $S_{e}$ and $S_{e}^{*}$, respectively, such that

$$
\langle J \varphi, \mu\rangle_{\partial \widehat{\Omega}}=\langle\varphi, \mu\rangle_{\partial \widehat{\Omega}} \quad \forall \mu \in S_{e}^{*}
$$

and

$$
\left\langle J^{*} \varphi, \chi\right\rangle_{\partial \widehat{\Omega}}=\langle\varphi, \chi\rangle_{\partial \widehat{\Omega}} \quad \forall \chi \in S_{e}
$$

We observe that $J$ and $J^{*}$ are dual operators with respect to the inner product on $L^{2}(\partial \widehat{\Omega})$, and it follows from (7.2) that

$$
|J \varphi|_{\partial \widehat{\Omega}},\left|J^{*} \varphi\right|_{\partial \widehat{\Omega}} \leq \alpha_{0}^{-1}|\varphi|_{\partial \widehat{\Omega}} .
$$

Hence, the operators $J$ and $J^{*}$ are stable in $L^{2}(\partial \widehat{\Omega})$.

However, in order to derive the desired properties of the operator $\widehat{\Pi}$, we shall need a similar stability property for the operator $J$, or more precisely, for an extension of $J$, in $H^{-1 / 2}(\partial \widehat{\Omega})$. In order to derive this stability property, we observe that it follows from the construction of the spaces $S_{e}^{*}$ above that there is a constant $c$, independent of $h$, such that the following approximation property and inverse property hold:

$$
\inf _{\mu \in S_{e}^{*}}|\varphi-\mu|_{\partial \widehat{\Omega}} \leq c h|\varphi|_{1, \partial \widehat{\Omega}} \forall \varphi \in H^{1}(\partial \widehat{\Omega})
$$

and

$$
|\mu|_{1, \partial \widehat{\Omega}} \leq c h^{-1}|\mu|_{\partial \widehat{\Omega}} \quad \forall \mu \in S_{e}^{*} .
$$

From these properties we can easily derive that the operator $J^{*}$ is stable in $H^{1}(\partial \widehat{\Omega})$, i.e., there is a constant $c$, independent of $h$, such that

$$
\left|J^{*} \varphi\right|_{1, \partial \widehat{\Omega}} \leq c|\varphi|_{1, \partial \widehat{\Omega}} \quad \forall \varphi \in H^{1}(\partial \widehat{\Omega}) .
$$

In order to see this, let $\varphi \in H^{1}(\partial \widehat{\Omega})$ be arbitrary and let $\varphi^{*} \in S_{e}^{*}$ be the $H^{1}$ projection of $\varphi$. Then $\left|\varphi^{*}\right|_{1, \partial \widehat{\Omega}} \leq|\varphi|_{1, \partial \widehat{\Omega}}$. Furthermore, for any $\psi \in L^{2}(\partial \widehat{\Omega})$ and $\mu \in S_{e}^{*}$,

$$
\left\langle J^{*} \varphi-\varphi, \psi\right\rangle_{\partial \widehat{\Omega}}=\langle\varphi-\mu, J \psi-\psi\rangle_{\partial \widehat{\Omega}},
$$


and hence it follows from duality, (7.3), and (7.4) that $\left|J^{*} \varphi-\varphi^{*}\right|_{\partial \widehat{\Omega}} \leq c h|\varphi|_{1, \partial \widehat{\Omega}}$. Hence, by (7.5),

$$
\left|J^{*} \varphi\right|_{1, \partial \widehat{\Omega}} \leq c h^{-1}\left|J^{*} \varphi-\varphi^{*}\right|_{\partial \widehat{\Omega}}+\left|\varphi^{*}\right|_{1, \partial \widehat{\Omega}} \leq c|\varphi|_{1, \partial \widehat{\Omega}},
$$

which is the desired bound (7.6).

Note that by interpolation, (7.3) and (7.6) imply that

$$
\left|J^{*} \varphi\right|_{1 / 2, \partial \widehat{\Omega}} \leq c|\varphi|_{1 / 2, \partial \widehat{\Omega}} \forall \varphi \in H^{1 / 2}(\partial \widehat{\Omega}),
$$

and by duality we therefore obtain that

$$
|J \varphi|_{-1 / 2, \partial \widehat{\Omega}} \leq c|\varphi|_{-1 / 2, \partial \widehat{\Omega}} \forall \varphi \in H^{-1 / 2}(\partial \widehat{\Omega}) .
$$

This is the desired stability of $J$ in $H^{-1 / 2}(\partial \widehat{\Omega})$.

The construction of the operator $\widehat{\Pi}$ is based on a proper decomposition of elements in $H(\operatorname{div}, \widehat{\Omega})$ in a gradient vector and a divergence-free vector. Let $v \in H(\operatorname{div}, \widehat{\Omega})$ be arbitrary, and consider the boundary value problem

$$
\begin{aligned}
\Delta \varphi=\nabla \cdot v & \text { in } \widehat{\Omega}, \\
\nabla \varphi \cdot \hat{\nu}=m(v) & \text { on } \partial \widehat{\Omega},
\end{aligned}
$$

where $m(v)$ is the mean value of $v \cdot \hat{\nu}$ on $\partial \widehat{\Omega}$. This problem has a solution $\varphi$ which is uniquely determined up to constants. Furthermore, if $v^{1}=\nabla \varphi$, then $v^{1} \in\left(H^{1}(\widehat{\Omega})\right)^{2}$, and there is a constant $c$, independent of $v$, such that $\left\|v^{1}\right\|_{1} \leq c\|\nabla \cdot v\|$, where $\|\cdot\|_{1}$ denotes the norm on $H^{1}(\widehat{\Omega})$ (or $\left.\left(H^{1}(\widehat{\Omega})\right)^{2}\right)$.

Hence, as in [16] we can define an element $\widehat{\Pi}^{1} v \in \widehat{V}$ by reproducing the average values of the normal components of $v^{1}$ on each edge of the squares of the grid. By construction, the function $\widehat{\Pi}^{1} v$ has the property that

$$
\hat{b}\left(\widehat{\Pi}^{1} v-v, q\right)=0 \quad \forall q \in \widehat{Q}
$$

and

$$
\widehat{\Pi}^{1} v \cdot \hat{\nu}=m(v) \quad \text { on } \partial \widehat{\Omega} .
$$

Furthermore, there exists a constant $c$, independent of $v$, such that

$$
\left\|\widehat{\Pi}^{1} v\right\|_{\text {div }} \leq c\|v\|_{\text {div }} .
$$

The function $\widehat{\Pi}^{1} v$ approximates the gradient part of the vector function $v$. We also need an approximation of the divergence-free component of $v$. Let $\psi \in H^{-1 / 2}(\partial \widehat{\Omega})$ be given by $\psi-v \cdot \hat{\nu}-m(v)$. Then

$$
\langle\psi, 1\rangle_{\partial \widehat{\Omega}}=\langle J \psi, 1\rangle_{\partial \widehat{\Omega}}=0 .
$$

Therefore, there exists an element $\eta \in Z_{B}$, uniquely determined up to constants, such that $\eta_{t}=J \psi$, where, as above, $\eta_{t}$ denotes the tangential derivative of $\eta$ in a counterclockwise direction. Furthermore, let $r \in Z$ be the solution of the discrete Poisson equation defined by the conforming finite element method:

Find $r \in Z$ such that

$$
\begin{aligned}
(\nabla r, \nabla z)=0 & \forall z \in Z \\
\left.r\right|_{\partial \widehat{\Omega}}=\eta & \text { on } \partial \widehat{\Omega}
\end{aligned}
$$


This problem has a unique solution. Furthermore, we can argue as in [7] (cf. also Lemma 3.2 of [6]) and conclude that $\|r\|_{1} \leq c|\eta|_{1 / 2, \partial \widehat{\Omega}}$, where the constant $c$ is independent of $\eta$. Observe also that the trace inequality $|\psi|_{-1 / 2, \partial \hat{\Omega}} \leq$ $c\|v\|_{\text {div }}$ holds. Hence, it follows from (7.7) and the fact that the map $\eta \mapsto \eta_{t}$ is continuous from $H^{1 / 2}(\partial \widehat{\Omega})$ to $H^{-1 / 2}(\partial \widehat{\Omega})$ that $\|r\|_{1} \leq c\|v\|_{\text {div }}$.

Define now $\widehat{\Pi}^{2} v=\left(r_{y},-r_{x}\right)^{T}$. Then, since $\nabla \cdot\left(\widehat{\Pi}^{2} v\right)=0$, it follows that

$$
\left\|\widehat{\Pi}^{2} v\right\|_{\text {div }} \leq c\|v\|_{\text {div }} .
$$

Since $\widehat{\Pi}^{2} v$ is divergence-free, it is also obvious that

$$
\hat{b}\left(\widehat{\Pi}^{2} v, q\right)=0 \quad \forall q \in \widehat{Q} .
$$

Finally, for any $\mu \in S^{*}$,

$$
\begin{aligned}
\left\langle\mu,\left(\widehat{\Pi}^{2} v-v\right) \cdot \hat{\nu}\right\rangle_{\widehat{\Gamma}} & =\left\langle\mu_{e}, \widehat{\Pi}^{2} v \cdot \hat{\nu}-\psi\right\rangle_{\partial \widehat{\Omega}}-\left\langle\mu_{e}, m(v)\right\rangle_{\partial \widehat{\Omega}} \\
& =-\langle\mu, m(v)\rangle_{\widehat{\Gamma}},
\end{aligned}
$$

where, as above, $\mu_{e}$ is the zero extension of $\mu$. The desired operator $\widehat{\Pi}$ is now defined by letting $\widehat{\Pi} v=\widehat{\Pi}^{1} v+\widehat{\Pi}^{2} v$. The uniform boundedness of these operators follows from (7.11) and (7.13), and the desired property (7.1) follows from (7.9), (7.10), (7.14), and (7.15).

In order to complete the construction of a Dirichlet preconditioner as studied in $\S 6$, we also need an operator $\Lambda: S^{*} \mapsto S^{*}$, symmetric with respect to the inner product on $L^{2}(\Gamma)$, such that the bounds $(6.15)$ hold. However, since $S^{*} \subset H_{0}^{1}(\Gamma)$ is a space consisting of piecewise linear functions with respect to a uniform partition, we can adopt the construction given in [6] and use the "square root of the discrete second derivative along $\Gamma$." Furthermore, as described in [6], equations of the form (6.18) can be solved by the Fast Fourier Transform.

In all the calculations, $g=0$, i.e., the elliptic equation has homogeneous Dirichlet boundary conditions. Furthermore, $f(x)=2$, and the initial approximation in the minimum residual method is set to zero. The iteration is terminated when the norm of the residual, induced by the inner product (4.4), is reduced by a factor of $10^{-5}$.

Example 7.1. In the first example we solve the Poisson problem, i.e., we choose $K(x)$ to be the identity matrix. The number of iterations, with and without preconditioning, are listed in Table 7.1. These results seem to confirm that the Dirichlet preconditioner results in a linear system where the number of iterations required by the minimum residual method is independent of $h$. Furthermore, the application of the Neumann preconditioner reduces the number of iterations considerably. Also, as expected from the analysis given in $\S 3$, the increase in the number of iterations, when the mesh parameter $h$ decreases, is slower when the Neumann preconditioner is applied than without a preconditioner.

Example 7.2. In the next example we consider a variable-coefficient problem with the matrix $K(x)$ given by

$$
K(x)=\left(\begin{array}{cc}
1+4\left(x_{1}^{2}+x_{2}^{2}\right) & 3 x_{1} x_{2} \\
3 x_{1} x_{2} & 1+11\left(x_{1}^{2}+x_{2}^{2}\right)
\end{array}\right) .
$$


TABLE 7.1. Number of iterations for the Poisson example

\begin{tabular}{|c|c|c|c|c|}
\hline$h$ & $1 / 16$ & $1 / 32$ & $1 / 64$ & $1 / 128$ \\
\hline \# it. no preconditioner & 75 & 139 & 271 & 544 \\
\hline \# it. Neumann preconditioner & 14 & 18 & 22 & 30 \\
\hline \# it. Dirichlet preconditioner & 10 & 12 & 12 & 12 \\
\hline
\end{tabular}

In this example the condition number of the matrix $A$, corresponding to the bilinear form $a(\cdot, \cdot)$, is larger than in the previous example, but still independent of the mesh parameter $h$. We therefore expect the minimum residual method to use more iterations to converge than in the previous example. However, the dependence of the convergence rate on $h$ should be comparable with the previous Poisson example. Table 7.2 seems to indicate that this is also the case.

TABLE 7.2. Number of iterations for the variable-coefficient example

\begin{tabular}{|c|c|c|c|c|}
\hline$h$ & $1 / 16$ & $1 / 32$ & $1 / 64$ & $1 / 128$ \\
\hline \# it. no preconditioner & 174 & 325 & 627 & 1323 \\
\hline \# it. Neumann preconditioner & 71 & 86 & 104 & 138 \\
\hline \#it. Dirichlet preconditioner & 62 & 68 & 71 & 72 \\
\hline
\end{tabular}

We note that the performance of our method depends only on the conditioning of the involved operators, not on the structure of $A$. We use exactly the same preconditioner in Example 7.1 and Example 7.2, and the work in each iteration is virtually the same. In contrast, a method requiring the inversion of $A$, or the exact inversion of the given problem on the subdomains, will require much more work for the variable-coefficient problems in Example 7.2 than for the Poisson problem in Example 7.1. This is because the nondiagonal form of the matrix $K$ will make the band structure of the matrix $A$ more complex.

\section{BIBLIOGRAPHY}

1. O. Axelsson and V. A. Barker, Finite element solution of boundary value problems. Theory and computation, Academic Press, Orlando, FL, 1984.

2. K. Aziz and A. Settari, Petroleum reservoir simulation, Appl. Sci. Publ., London, 1979.

3. P. E. Bjørstad and O. B. Widlund, Iterative methods for the solution of elliptic problems on regions partitioned into substructures, SIAM J. Numer. Anal. 23 (1986), 1097-1120.

4. J. H. Bramble, R. E. Ewing, J. E. Pasciak, and A. H. Schatz, A preconditioning technique for the efficient solution of problems with local grid refinement, Comput. Methods Appl. Mech. Engrg. 67 (1988), 149-159.

5. J. H. Bramble and J. E. Pasciak, A preconditioned technique for indefinite systems resulting from mixed approximations of elliptic problems, Math. Comp. 50 (1988), 1-17.

6. J. H. Bramble, J. E. Pasciak, and A. H. Schatz, The construction of preconditioners for elliptic problems by substructuring. I, Math. Comp. 47 (1986), 103-134.

7. Math. Comp. 46 (1986), 361-369.

8. F. Brezzi, On the existence, uniqueness and approximation of saddle-point problems arising from Lagrangian multipliers, RAIRO Numer. Anal. 8 (1974), 129-151. 
9. F. Brezzi, J. Douglas, Jr., R. Duràn, and M. Fortin, Mixed finite elements for second order elliptic problems in three variables, Numer. Math. 51 (1987), 237-250.

10. F. Brezzi, J. Douglas, Jr., M. Fortin, and L. D. Marini, Efficient rectangular mixed finite elements in two and three space variables, RAIRO Modél. Math. Anal. Numér. 21 (1987), 581-604.

11. F. Brezzi, J. Douglas, Jr., and L. D. Marini, Two families of mixed finite elements for second order elliptic problems, Numer. Math. 47 (1985), 217-235.

12. T. F. Chan and H. C. Elman, Fourier analysis of iterative methods for elliptic problems, SIAM Rev. 31 (1989), 20-49.

13. J. Douglas, Jr., R. E. Ewing, and M. F. Wheeler, The approximation of the pressure by a mixed method in the simulation of miscible displacement, RAIRO Numer. Anal. 17 (1983), 17-23.

14. J. Douglas, Jr. and P. Pietra, A description of some alternating-direction iterative techniques for mixed finite element methods, Mathematical and Computational Methods in Seismic Exploration and Reservoir Modeling (W. E. Fitzgibbon, ed.), SIAM, Philadelphia, PA, 1986, pp. 37-53.

15. R. E. Ewing and M. F. Wheeler, Computational aspects of mixed finite element methods, Numerical Methods for Scientific Computing (R. S. Stepleman, ed.), North-Holland, Amsterdam, 1983, pp. 163-172.

16. M. Fortin, An analysis of the convergence of mixed finite element methods, RAIRO Numer. Anal. 11 (1977), 341-354.

17. M. Fortin and R. Glowinski, Augmented Lagrangian methods: Applications to the numerical solution of boundary value problems, North-Holland, Amsterdam, 1983.

18. V. Girault and P. A. Raviart, Finite element methods for Navier-Stokes equations, SpringerVerlag, Berlin, 1986.

19. R. Glowinski and M. F. Wheeler, Domain decomposition and mixed finite element methods for elliptic problems, Proc. 1st Internat. Sympos. on Domain Decomposition Methods for Partial Differential Equations (R. Glowinski, G. H. Golub, G. A. Meurant, and J. Periaux, eds.), SIAM, Philadelphia, PA, 1988, pp. 144-172.

20. J. L. Lions and E. Magenes, Non-homogeneous boundary value problems and applications, vol. I, Springer, New York, 1972.

21. P. L. Lions, On the Schwarz alternating method, Proc. 1st Internat. Sympos. on Domain Decomposition Methods for Partial Differential Equations (R. Glowinski, G. H. Golub, G. A. Meurant, and J. Periaux, eds.), SIAM, Philadelphia, PA, 1988, pp. 1-42.

22. T. P. Mathew, Domain decomposition and iterative refinement methods for mixed finite element discretizations of elliptic problems, Ph.D. thesis, Department of Computer Science, Courant Institute of Mathematical Sciences, 1989.

23. J. .4. Meijerink and H. A. van der Vorst, An iterative solution method for linear systems of which the coefficient matrix is a symmetric M-matrix, Math. Comp. 31 (1977), 148-162.

24. C. C. Paige and M. A. Saunders, Solution of sparse indefinite systems of linear equations, SIAM J. Numer. Anal. 12 (1975), 617-629.

25. P. A. Raviart and J. M. Thomas, A mixed finite element method for 2-nd order elliptic problems, Mathematical Aspects of Finite Element Methods (I. Galligani and E. Magenes, eds.), Lecture Notes in Math., vol. 606, Springer-Verlag, Berlin, 1977, pp. 292-315.

26. T. F. Russel and M. F. Wheeler, Finite element and finite difference methods for continuous flow in porous media, The Mathematics of Reservoir Simulation (R. E. Ewing, ed.), SIAM, Philadelphia, PA, 1983.

27. T. Rusten and R. Winther, A preconditioned iterative method for saddlepoint problems, SIAM J. Matrix Anal. Appl. 13 (1992), 887-904.

Department of Mathematics, University of Oslo, P.O. Box. 1080 Blindern, N-0316 OSLO 3, NORWAY

E-mail address: torgeir@ifi.uio.no

E-mail address: ragnar@ifi.uio.no 